\title{
Diferenças raciais entre pacientes com esclerose glomerular focal e glomerulonefrite membranoproliferativa residentes no estado da Bahia
}

\author{
A. A. Lopes, R. P. Martinelli, M. A. Silveira, H. Rocha \\ Trabalho realizado no Hospital Universitário Prof. Edgard Santos, Universidade Federal da Bahia, Salvador, BA
}

\begin{abstract}
RESUMO - Ов етіvo. Avaliar a relação entre raça e tipo histológi co de glomerulonefrite, levando em consideração idade, sexo e presença da forma hepatoesplênica da esquistossomose mansônica.

Material e Métodos. Pacientes do Serviço de Nefrologia da Universidade Federal da Bahia, 80 com esclerose glomerular focal (EGF) e $50 \mathrm{com}$ glomerulonefrite membranoproliferativa (GNMP) foram comparados quanto à distribuição dos tipos raciais (negro, mulato, branco). Pacientes com lupus eritematoso sistêmico ou qualquer outro tipo de doença auto-imune não foram incluídos na presente análise. Comparações ajustadas foram feitas através do método de Mantel-Haenszel e de um modelo de regressão logística múltipla.

Resultados. Raça foi significantemente associada com o tipo histológico; a relação entre o número de negros ou mulatos e o número de brancos foi aproximadamente 2,4 vezes maior (odds ratio=2,43; IC $95 \%=1,09-5,45$ ) em pacientes
\end{abstract}

\section{NTRODUÇÃO}

A distribuição dos diversos tipos de glomerulonefrites em material de biópsia renal varia na dependência do local do estudo1-4. Um trabalho realizado na Bahia mostrou uma predominância dos tipos histológicos esclerose glomerular focal (EGF) e glomerulonefrite membranoproliferativa (GNMP) em pacientes biopsiados. ${ }^{4} \mathrm{~A}$ freqüência de glomerul onefrite membranosa foi relativamente baixa, particularmente ao se levar em consi deração que este tipo histológico é um dos mais freqüentes em pacientes adultos biopsiados nos Estados Unidos e Europa. Um outro fator que pode contribuir para a freqüência relativamente el evada de EGF e GNMP na Bahia é a alta prevalência da forma hepatoesplênica da esquistossomose mansônica ${ }^{5-9}$.

E m adi ção ao l ocal, outras características demográficas, incluindo fatores raciais, podem também influenciar a prevalência de determinados tipos histológicos de glomerulonefrites. Estudos realizados nos Estados Unidos, por exemplo, mostram uma maior freqüência de EGF em negros ${ }^{10-12}$. As razões para esta diferença racial em EGF, no com EGF do que em pacientes com GNMP. Esta associação entre raça e tipo histológico foi independente da idade, do sexo e da presença da forma hepatoesplênica da esquistossomose. No modelo de regressão logística múltipla, raça foi significativamente $(p=0,037)$ associada com o tipo histológico (odds ratio=2,54; IC $95 \%=1,06-6,06$ ).

Conclusão. A maior freqüência de negros e mulatos no grupo EGF nesta amostra de pacientes da Bahia é consistente com os achados de estudos realizados nos Estados Unidos. Os dados apoiam a possibilidade de uma relação entre descendência africana e susceptibilidade aumentada para EGF, independente da idade, do sexo e do diagnóstico de esquistossomose. A identificação dos mecanismos que determinam esta diferença racial representa uma importante questão para futuras investigações.

UnITERMOS: Raça. Negros. Glomerulonefrite. Esquistossomose.

entanto, permanecem não esclarecidas. O presente estudo avalia possíveis diferenças na distribuição racial entre pacientes com EGF e GN MP residentes no estado da Bahia, levando em consideração o sexo, a idade, bem como a presença e a forma clínica, hepatoesplênica (HEME) ou hepatointestinal $(\mathrm{HI})$, da esquistossomose.

\section{MÉ TODOS}

Pacientes com EGF $(\mathrm{N}=80)$ foram comparados com portadores de GNMP $(\mathrm{N}=50)$ no que se refere às características iniciais, definidas de acordo com informações registradas nos prontuários médicos. Embora as informações tenham sido analisadas transversalmente, os pacientes estudados fazem parte de um estudo de coorte que foi desenhado com o objetivo primário de avaliar fatores de risco para o desenvolvimento de doença renal terminal. Todos os pacientes incluídos no estudo foram, ou estão sendo, acompanhados no Hospital Universitário Prof. Edgard Santos da Universidade Federal da Bahia; as biópsias renais foram analisadas no Serviço de Anatomia Patológica do mesmo hospital, 
Tabela 1 - Distribuição das características demográficas, diagnóstico e forma clínica da esquitossomose de acordo com o tipo histológico (esclerose glomerular focal vs glomerulonefrite membranoproliferativa)

\begin{tabular}{|c|c|c|c|c|}
\hline \multirow[b]{3}{*}{ Idade em anos } & \multicolumn{2}{|c|}{ Tipos Histológicos* } & \multirow[b]{2}{*}{$\mathbf{O R}^{\Phi}$} & \multirow{3}{*}{$\begin{array}{c}\text { VALOR } \\
\text { de } p\end{array}$} \\
\hline & EGF & GNMP & & \\
\hline & $\mathrm{N}=80$ & $\mathrm{~N}=50$ & IC 95\% & \\
\hline Idade (média $\pm D P)$ & $20,2 \pm 13,7$ & $31,0 \pm 12,7$ & & $<0,001$ \\
\hline mediana & 17,0 & 30,9 & & $<0,001$ \\
\hline$\%$ ldade $>18$ anos & 47,5 & 90,0 & $0,10(0,03-0,29)$ & $<0,001$ \\
\hline \multicolumn{5}{|l|}{ Sexo } \\
\hline$\%$ Masculino & 55,0 & 70,0 & $0,52(0,23-1,17)$ & $0,127^{\psi}$ \\
\hline \multicolumn{5}{|l|}{ Raça } \\
\hline$\%$ Negros e Mulatos & 72,5 & 52,0 & $2,43(1,09-5,45)$ & $0,029^{\psi}$ \\
\hline \multicolumn{5}{|l|}{ Esquistossomose } \\
\hline Ausente & 30,7 & 30,0 & & $0,002^{\psi}$ \\
\hline $\mathrm{HI}^{\downarrow}$ & 40,0 & 14,0 & & \\
\hline $\mathrm{HEME}^{\uparrow}$ & 29,3 & 56,0 & & \\
\hline \multicolumn{5}{|c|}{ * EGF = Esclerose Glomerular Focal, $\mathrm{GNMP}=$ glomerulonefrite membranoproliferativa } \\
\hline \multicolumn{5}{|c|}{$\Phi$ OR = Odds Ratio } \\
\hline \multicolumn{5}{|c|}{ ळ IC = Intervalo de Confiança } \\
\hline \multicolumn{5}{|c|}{$\psi$ Teste Exato de Fisher } \\
\hline \multicolumn{5}{|c|}{$\downarrow \mathrm{HI}=$ Forma Hepatointestinal da Esquitossomose } \\
\hline \multicolumn{5}{|c|}{$\uparrow \quad \mathrm{HEME}=$ Forma Hepatoesplênica da Esquitossomose } \\
\hline
\end{tabular}

entre 1969 e 1994. Nenhum dos pacientes selecionados teve o diagnóstico de lupus eritematoso sistêmico ou qualquer outro tipo de doença autoimune. Os pacientes foram classificados como brancos, mulatos ou negros. Com o objetivo de aumentar o poder estatístico do estudo, em algumas análises, mulatos e negros foram incluídos na mesma categoria. Foi considerada a idade do paciente na época da primeira consulta nefrológica. O diagnóstico e a definição da forma da esquistossomose (hepatointestinal ou hepatoesplênica) foram baseados no parasitológico de fezes e na pal pação esplênica.

O teste exato de Fisher foi usado para avaliar a significância estatística de associações entre o tipo histológico e variáveis demográficas categóricas (i.e., raça, sexo e idade). Neste tipo de análise a idade foi categorizada em dois níveis, $\leq 18$ e $>18$ anos. A idade foi também usada como variável contínua, sendo as diferenças de médias entre os grupos avaliadas através do teste $t$ de Student após transformação logarítmica dos val ores originais, em conformidade com a premissa de distribuição normal.

F oram desenvolvidos modelos de regressão logística para avaliar a associação entre raça e tipo histológico, ajustada para potenciais influências de co-variáveis. A necessidade de modelos logísticos estratificados por níveis de determinados fatores foi avaliada através dos coeficientes de interação entre pares de variáveis ${ }^{13,14}$. Foram defini das duas variáveis indi cadoras (dummy variables) com o objetivo de avaliar a influência da presença e da forma clínica da esquistossomose mansônica na associação entre raça e tipo histológico.

Os procedimentos descritivos, as comparações de médias e a regressão logística foram realizados através do software Statistical Package for Social Science- SPSS ${ }^{15,16}$. O procedimento NPAR do SPSS foi usado para testar diferenças de medianas. O módulo CaseCont do "Computer Programs for Epidemiologic Analysis", versão 2,5, foi usado para determinar 1) odds ratios ajustadas para os potenciais efeitos de co-variáveis, através da técnica de Mantel-Haenszel, 2) testes de homogeneidade entre odds ratios e 3 ) interval os de confiança por um método exato ${ }^{17}$. Através do módulo Exact2xk do mesmo software foram determinados os níveis de significância estatística das associações entre variáveis categóricas. ${ }^{17}$

\section{RESULTADOS}

As características demográficas de acor do com o tipo histológico são mostradas na Tabela 1 . As médias $(20,2 \pm 13,7$ vs $31,0 \pm 12,7$ anos) e medianas (17,0 vs 30,9 anos) de idade foram significantemente menores no grupo com EGF. A proximadamente $47,5 \%$ dos pacientes com E GF e $90 \%$ dos com GNMP tinham idades superiores a 18 anos (odds 
Tabela 2 - Estimativas da associação entre raças (negros e mulatos vs brancos) e tipo de glomerulonefrite* (EGF vs GNMP) ajustadas para o sexo, idade e esquistossomose

\begin{tabular}{|c|c|c|c|c|c|c|c|c|}
\hline \multirow{4}{*}{$\begin{array}{l}\text { Negros+Mulatos } \\
\text { Brancos }\end{array}$} & \multicolumn{3}{|c|}{ Masculino } & \multicolumn{3}{|c|}{ Feminino } & \multicolumn{2}{|c|}{$\begin{array}{c}\text { Ajustada para Sexo } \\
\text { através } \mathrm{MH}^{\lambda}\end{array}$} \\
\hline & $\begin{array}{l}\text { EGF } \\
(\mathrm{N})\end{array}$ & $\begin{array}{l}\text { GNMP } \\
(\mathrm{N})\end{array}$ & $\begin{array}{c}\text { OR }^{\Phi} \\
(\text { IC 95\%) }\end{array}$ & EGF & GNMP & $\begin{array}{c}\text { OR } \\
\text { (IC 95\%) }\end{array}$ & $\begin{array}{c}\mathrm{OR}_{\mathrm{MH}}{ }^{\xi} \\
(\mathrm{IC} 95 \%)\end{array}$ & Valor de $p^{\psi}$ \\
\hline & 31 & 18 & 2,25 & 27 & 8 & 2,63 & 2,37 & \\
\hline & 13 & 17 & $(0,81-6,31)$ & 9 & 7 & $(0,61-11,04)$ & $1,05-5,31$ & 0,037 \\
\hline Negros+Mulatos & \multicolumn{6}{|c|}{$>18$ anos } & \multicolumn{2}{|c|}{$\begin{array}{l}\text { Ajustada para Idade } \\
\text { através MH }\end{array}$} \\
\hline Brancos & $\begin{array}{l}\text { EGF } \\
(\mathrm{N})\end{array}$ & $\begin{array}{l}\text { GNMP } \\
(\mathrm{N})\end{array}$ & $\begin{array}{c}\text { OR } \\
\text { (IC 95\%) }\end{array}$ & EGF & GNMP & $\begin{array}{l}\text { OR } \\
\text { (IC 95\%) }\end{array}$ & $\begin{array}{c}\mathrm{OR}_{\mathrm{MH}} \\
(\mathrm{IC} 95 \%)\end{array}$ & Valor de $p$ \\
\hline & 30 & 2 & 3,75 & 28 & 24 & 2,45 & 2,65 & \\
\hline & 12 & 3 & $0,37-48,64$ & 10 & 21 & $(0,89-6,99)$ & $(1,07-6,72)$ & 0,034 \\
\hline \multicolumn{9}{|c|}{$\begin{array}{c}\text { Ajustada para Esquistossomose } \\
\text { através MH }\end{array}$} \\
\hline \multirow[t]{3}{*}{ Brancos } & $\begin{array}{l}\text { EGF } \\
(\mathrm{N})\end{array}$ & $\begin{array}{l}\text { GNMP } \\
(\mathrm{N})\end{array}$ & $\begin{array}{c}\text { OR } \\
\text { (IC 95\%) }\end{array}$ & EGF & GNMP & $\begin{array}{c}\text { OR } \\
\text { (IC 95\%) }\end{array}$ & $\begin{array}{c}\mathrm{OR}_{\mathrm{MH}} \\
(\mathrm{IC} 95 \%)\end{array}$ & valor de $p$ \\
\hline & 42 & 12 & 2,19 & 16 & 14 & 2,67 & 2,38 & \\
\hline & 16 & 10 & $0,69-6,78$ & 6 & 14 & $(0,70-10,73)$ & $(1,02-5,48)$ & 0,044 \\
\hline \multicolumn{9}{|c|}{$\begin{array}{ll}* & \text { EGF = Esclerose Glomerular Focal, GNMP=glomerulonefrite me } \\
\lambda & \mathrm{MH}=\text { Técnica de Mantel Haenszel } \\
\Phi & \mathrm{OR}=\text { Odds Ratio Específica do Estrato } \\
\varpi & \mathrm{IC}=\text { Intervalo de Confiança } \\
\xi & \mathrm{OR}_{\mathrm{MH}}=\text { Odds Ratio ajustada pela Técnica de Mantel-Haenszel } \\
\psi & \text { Teste Exato de Fisher } \\
\downarrow & \mathrm{HI}=\text { Forma Hepatointestinal da Esquitossomose } \\
\uparrow & \text { Hepato -Esplenomegalia Esquistossomótica }\end{array}$} \\
\hline
\end{tabular}

ratio $(O R)=0,1 ;$ IC95\%=0,04-0,29). A freqüência de indi víduos do sexo masculino foi também menor no grupo com EGF (55\% vs $70 \%$; OR $=0,52$; IC95\% $=0,23-1,17 ; p=0,127)$. A freqüência de mulatos e negros, em conjunto, foi significativamente maior $(p=0,029)$ no grupo com E GF $(72,5 \%$ vs $52 \%)$ do que no grupo com GNMP. A relação entre o número de pacientes negros ou mulatos e o número de pacientes brancos foi aproximadamente 2,4 vezes maior (odds rati o=2,43; I C $95 \%=1,09-5,45$ ) no grupo E GF do que no grupo GN MP. E mbora a prevalência de esquistossomose tenha sido semelhante entre os tipos histológicos, o percentual de pacientes com a forma hepatoesplênica foi menor em pacientes com E GF $(29,3 \%)$ do que nos pacientes com GN MP (56\%). No conjunto, a associ ação entre ti po histológico e esquistossomose foi estatisticamente significante $(p=0,002)$.

A Tabela 2 mostra as estimativas pontuais e os I C95\% da associação entre raça e tipo histológico de acordo com sexo, idade e diagnóstico de esquistossomose. As estimativas ajustadas (ORs $=2,37$; 2,65 e 2,38) através da técnica de Mantel-Haenszel foram estatisticamente significantes $(p<0,05)$ e semelhantes à estimativa não ajustada $(O R=2,43)$ mostrada na Tabela 1. Além do mais, esta associação entre raça e tipo histol ógi co não foi modificada de forma importante pelas variáveis idade, sexo e esquistossomose (ausente ou hepatointestinal vs hepatoesplênica). Todos os testes de homogeneidade foram estatisticamente não significantes $(p>0,1)$.

E stimativas das associações entre o tipo histológico e as variáveis demográficas, ajustadas através de regressão logística, são mostradas na Tabela 3. A associação entre raça e tipo histológico praticamente não se alterou após o ajuste para as potenciais influências da idade, sexo e tipo histológico. No model o logístico, raça (negro ou mulato vs branco: OR=2,54; I C $95 \%=1,06-6,06$ ) e 


\begin{tabular}{|c|c|c|c|}
\hline Característica & $\mathbf{O R}^{\Phi}$ & IC 95\% & Valor de $p$ \\
\hline Raça (negro + mulatos vs brancos) & 2,54 & $(1,06-6,06)$ & 0,037 \\
\hline Sexo (masculino vs feminino) & 0,65 & $(0,27-1,58)$ & 0,348 \\
\hline Idade (>18 anos vs $£ 18$ ) & 0,13 & $(0,04-0,38)$ & $<0,001$ \\
\hline \multicolumn{4}{|c|}{$\begin{array}{l}\text { * O modelo de regressão logística múltipla incluiu raça ( } 1=\text { mulato ou negro, } 0=\text { branco }) \text {, sexo }(1=\text { masculino, } 0=\text { feminino }) \text { idade }(1=\text { superior } \\
\text { a } 18 \text { anos, } 0=\text { outras idades) e duas variáveis indicadoras (dummy), uma para a forma hepatointestinal }(1=\operatorname{sim}, 0=\text { não) e outra para a forma } \\
\text { hepatoesplênica ( } 1=\operatorname{sim}, 0=\text { não). A variável resposta, tipo histológico, foi codificada como } 1=E G F \text { e } 0=G N M P \text {. } \\
\Phi \text { OR =odds ratio }\end{array}$} \\
\hline
\end{tabular}

idade ( $>18$ vs $\leq 18$ anos: OR $=0,13$; IC $95 \%=0,04$ 0,38 ) foram significativamente associadas com tipo histológico (EGF vs GNMP)

\section{DISCUSSÃO}

A maior freqüência de negros e mulatos no grupo EGF do que no grupo GNMP, mostradas no presente trabalho, é consistente com o que tem sido relatado em estudos realizados nos Estados Unidos ${ }^{10-12}$. É importante observar que apesar do tipo histológico estar também relacionado com a idade e o sexo, a associação da raça com o diagnóstico histológico foi similar entre homens e mulheres e entre os grupos etários. Os resultados apresentados estão de acordo com a possibilidade de que pessoas negras e mulatas do estado da Bahia, similarmente aos negros americanos, têm uma mai or predisposição para EGF do que as pessoas brancas.

Os dados do presente estudo podem ser vistos como mais uma evidência de um possível papel de fatores ligados com descendência africana na predisposição para EGF. Alguns avanços no conhecimento sobre a patogênese das doenças glomerulares podem ajudar a identificar os verdadeiros fatores que determinam a diferença racial em EGF. Existem indícios, por exemplo, de que hipertensão intraglomerular é um dos mecanismos que propiciam o desenvolvimento de E GF ${ }^{18-19}$. Ao lado disso, importantes di ferenças entre negros e brancos no perfil hemodinâmico renal têm sido observadas $^{20-21}$. J á foi mostrado que, após a ingestão de quantidades moderadas de sódio, a taxa de filtração glomerular se el eva mais no negro do que no branco americano ${ }^{21}$. Esse fenômeno parece estar associado com uma maior reabsorção tubular de sódio em pessoas de descendência africana, uma característica que pode ser até mais marcante em negros e miscigenados que nasceram no Brasil e nos E stados U nidos do que em negros africanos ${ }^{22,23}$. Existem também evidências de que distúrbios no funcionamento da célula mesangial contribuem para EGF ${ }^{18}$. É possível que os negros e os mulatos apresentem alterações mesangiais que resultem em maior produção de teci do fibroso mais freqüentemente do que os brancos. Dustan ${ }^{24}$ tem sugerido que a mai or predisposição do negro para esclerose renal pode ser mediada por mecanismos semeIhantes aos que determinam a sua maior predisposição para quelóides ${ }^{25}$. Como se sabe, a característica básica do quelóide é uma intensa proliferação de células fibroblásticas, presumivelmente em resposta à fatores de proliferação tissular ${ }^{26,27}$.

$\mathrm{O}$ achado de que a associ ação entre raça e EGF independe da idade, apoia a idéi a de que a diferença racial na distribuição de tipos histológi cos pode ser mediada por fatores já presentes na vida uterina. Lopes e Port ${ }^{28,29}$, após anal isarem os resultados de pesquisas básicas e de estudos epidemiológicos observacionais, concluíram que era plausível a hipótese (the low birth weight hypothesis) de que a maior freqüência de recém-nascidos com baixo peso no negro americano (comparado com o branco americano) poderia explicar a sua maior predisposição para nefropatias e doença renal terminal. Existem evidências de que o baixo peso ao nascer se associa com a redução do número de néfrons ${ }^{30}$, uma alteração que presumivelmente contribui para esclerose glomerular através do aumento da pressão intraglomerular ${ }^{31}$. Além do mais, a redução do número de néfrons pode ativar a produção de fatores de proliferação tissular, podendo, dessa forma, levar a um acúmulo de matriz mesangial e propiciar o desenvolvimento de esclerose glomerular ${ }^{32}$.

É importante observar que, no presente trabaIho, a associação entre raça e tipo histológico foi ajustada para o diagnóstico e forma clínica da esquistossomose. A associação ajustada foi seme- 
Ihante à não-ajustada, mostrando que a maior freqüência de negros e mulatos no grupo com E GF não dependeu da presença ou da forma de apresentação da esquistossomose. Esse é um achado importante, particularmente se forem consideradas as evidências de que o negro tem maior resistência para a fibrose hepática da esquistossomose ${ }^{33,34}$. Essa resistência para HEME no negro pode estar relacionada com um processo de seleção genética determinado pela exposi ção milenar dos africanos ao S. mansoni .

No presente estudo, a freqüência das formas hepatoesplênicas da esquistossomose foi maior no grupo com GNMP do que no grupo com EGF. I sto apoia um possível papel da maior resistência para o desenvolvimento de HEME em descendentes de africanos na predominância de negros e mulatos no grupo com EGF. É importante observar, no entanto, que houve um pequeno percentual de pessoas classificadas como negras na presente amostra, havendo um predomínio de mulatos. De acor do com estudos real izados em áreas endêmi cas de esquistossomose, a resistência para HEME em descendentes de africanos é reduzida com a miscigenação racial, sendo semel hante entre mulatos e brancos. ${ }^{34}$ I sto possivelmente explica porque, no presente estudo, a associação entre raça e tipo histológico não foi influenciada pela presença ou forma de apresentação da esquistossomose ${ }^{35}$.

Embora os achados que foram aqui mostrados sejam condizentes com os de outros estudos, o fato de que a classificação racial utilizada foi baseada em informações de prontuários pode ser motivo de questionamentos quanto a validade dessa variável. E mbora existam classificações raciais baseadas em critérios mais rigorosamente definidos e possíveis de serem realizadas diretamente pelo investigador no momento em que o paciente é entrevistado, não está cl aro se estas classificações são capazes de captar com maior fi delidade aspectos relacionados com a patogênese ou susceptibilidade para doenças. Além do mais, já foi mostrado que indivíduos classificados no prontuário médico como negros e mulatos são mais freqüentemente portadores de sobrenome de conotação religiosa, uma característica que tem sido relacionada com ancestralidade africana ${ }^{36}$.

E $m$ resumo, os dados do presente estudo sugerem uma predisposição de negros e mulatos para EGF, independente da idade, do sexo e da presença ou forma clínica da esquistossomose mansônica. Os resultados aqui mostrados, particularmente quando analisados em conjunto com os de outros estudos, apoiam a idéi a de que uma maior susceptibilidade para EGF em mulatos e negros possa ser mediada por fatores herdados de seus ancestrais africanos. As razões para uma mai or predisposição de negros e mulatos para EGF é uma importante questão que merece ser explorada em investigações futuras.

\section{AGRADE CIME NTOS}

Agradecemos a enfermeira Gildete Lopes e a Prof. Norma Lopes pelas revisões durante o desenvol vimento do artigo. Também agradecemos ao Serviço de Anatomia Patológica do Hospital Universitário Professor E dgard Santos pela ajuda no levantamento dos dados de biópsias renais. O Dr. Antonio Al berto Lopes é pesquisador bolsista do CNPq.

\section{SUMMARY}

\section{Racial differences between patients with focal segmental glomerulosclerosis and Membranoproliferative Glomerulonephritis from the State of Bahia}

OBJ ECTIVE. To assess the association between race and type of glomerulonephritis, taking into account age, gender and the presence of hepatosplenic schistosomiasis mansoni.

Methods. Patients from the Renal Service of the Federal University of Bahia, Brazil, 80 with focal segmental glomerulosclerosis (FSG) and 50 with membranoproliferative glomerulonephritis (MPGN) were compared regarding the distribution of the racial types (black, mulatto, white). Patients with systemic lupus erythematosus or any kind of autoimmune disease were not included in the present analysis. Adjusted comparisons were performed using the Mantel-Haenszel method and a multivariate logistic regression model.

RESULTS. Race was significantly associated with histologic type; the odds of being classified as black or mulatto were approximately 2.4 times higher (odds ratio=2.43; IC 95\%=1.09-5.45) in patients with FSG than in those with MPGN. The association between race and histologic type was not influenced by the potential effects of age, gender and hepatosplenic schistosomiasis. In the multivariate logistic regression model, race was significantly associated $(p=0.037)$ with type of glomerulonephritis (odds ratio=2.54; IC $95 \%=1.06-6.06)$.

Conclusion. A higher frequency of negroes and mulattoes in theFSG group (compared with MPGN) in this samplefrom the State of Bahia is consistent with findings of previous studies from the United States. The data support the possibility of a greater susceptibility to FSG among negroes and 
mulattoes, independently of age, gender and schistosomiasis. The identification of the mechanisms that determine this racial difference repre sents an important question for future investigations. [Rev Ass Med Brasil 1999; 45(2): 115-20.]

KEY WORDS: Race. Black. Glomerulonefritis. Schistosomiasis.

\section{REFERÊ NCIAS}

1. Mazzarolo Cruz HM, Cruz J, Silva AL J r, Saldanha LB, de Oliveira Penna D. Prevalence of adult primary glomerular diseases: retrospective analysis of 206 kidney biopsies. Rev Hosp Clin Fac Med São Paulo 1996; 51: 3-6.

2. Simon P, Ramée MP, Autuly V, Laruelle E, Charasse C, Cam $G$, Ang KS. E pidemiology of primary glomerular diseases in a French region. Variations according to period and age. Kidney Int 1994; 46: 1.192-8.

3. Stratta P, Segoloni GP, Canavese C, Sandri L, Mazzucco G, Roccatello D, Manganaro $M$, Vercellone $A$. Incidence of biopsy-proven primary glomerulonephritis in an I talian province. Am J Kidney Dis 1996; 27: 631-9.

4. Martinelli R, Lopes AA, Silveira MAS, Rocha H. Distribution of primary glomerular diseases in Bahia, Brazil: twenty-five year experience. Nephrology 1997 (suppl 1): S108.

5. Rocha H, Cruz T, Brito E, Susin M. Renal involvement in patients with hepatosplenic Schistosomiasis mansoni. Am J Trop Med Hyg 1976; 25: 108-115.

6. Andrade ZA, Rocha H. Schistosomal glomerulopathy. Kidney Int 1979; 16: 23-29.

7. Barreto ML. Geographical and socioeconomic factors relating to the distribution of Schistosoma mansoni infection in an urban area of northeast Brazil. Bull World Health Organ 1991; 69: 93-102.

8. Prata A, Bina J C, Barreto AC, Alecrim MG. Ensaio de controle da transmissão da esquistossomose pela oxamniquine em uma localidade hiperendêmica. Rev I nst Med Trop 1980; 22: 182-9.

9. Tavares-N etoJ , Prata A. F orma hepatosplênica da esquistossomose mansônica, em relação a composição racial e ao nível sócio-econômico, em Catolandia-Bahia. Rev Soc Bras Med Trop 1990; 23: 37-42.

10. Bakir AA, Bazilinski NG, Rhee HL, Ainis H, Dunea G. Focal segmental glomerulosclerosis. A common entity in nephrotic black adults. Arch Intern Med 1989; 149: 1.802-4.

11. Korbet SM, Genchi RM, Borok RZ, Schwartz MM. The racial prevalence of glomerular lesions in nephrotic adults. Am J Kidney Dis 1996; 27: 647-51.

12. Pontier PJ , Patel TG. Racial differences in the prevalenceand presentation of glomerular disease in adults. Clin Nephrol 1994; 42: 79-84.

13. Hosmer DW J r, Lemeshow S. Applied logisticregression.J ohn Wiley \& Sons, NewY ork, NY, 1989.

14. Kleinbaum DG, Kupper LL, Morgenstern H. Epidemiology Research: Principles and QuantitativeM ethods. Belmont, CA, Lifetime Learning Publications, 1982.

15. Norušis MJ . SPSS for Windows: Base system user's guide, release 6.0, Chicago, IL, SPPS Inc,1993.
16. Norušis MJ . SPSS for Windows: Advanced Statistics, release 6.0, Chicago, IL, SPSS Inc, 1993.

17. Gahlinger PM, Abramson J H. Computer Programs for Epidemiologic Analysis: PEPI. Stone Mountain, GA, USD Inc, 1995.

18. Schnaper HW, Robson AM. Nephrotic Syndrome: minimal changedisease, focal gl omerulosclerosis, and rel ated disorders. In: Schrier RW, Gottschalk CW (eds): Diseases of the Kidney, chap 105. Boston, MA, Little Brown, 1993, pp 1.731-84.

19. Simons J L, Provoost AP, Anderson S, RennkeHG, Troy J L, Brenner BM. Modulation of glomerular hypertension defines susceptibility to progressive glomerular injury. Kidney Int 1994; 46: 396-404.

20. Luft FC, Grim CE, Higgins J T J r, Weinberger MH. Differences in response to sodium administration in normotensive white and black subjects. J Lab Clin Med 1977; 90: 555-62.

21. Parmer RJ , Stone RA, Cervanka J H. Renal hemodinamics in essential hypertension: racial differences in responseto changes in dietary sodium. Hypertension. 1994; 24: 752-75.

22. Wilson TW, Grim CE. Biohistory and blood pressure differences in blacks today: A hypothesis. Hypertension 1991; 17(suppl I): I-122-I-128.

23. Lopes AA. Raça e Hipertensão Arterial. HiperAtivo 1996; 3:153-162.

24. Dustan HP. Growth factors and racial differences in severity of hypertension and renal diseases. Lancet 1992; 339: 1.339-40.

25. Datubo-Brown DD. Keloids: a review of the literature. $\mathrm{Br}$ J Plast Surg 1990;43:70-77.

26. Kikuchi K, Kadono T, Takehara K. Effects of various growth factors and histamine on cultured keloid fibroblasts. Dermatology 1995; 190: 4-8.

27. Bettinger DA, Yager DR, Diegelmann RF, Cohen IK. The effect of TGF-beta on keloid fibroblast proliferation and collagen synthesis. Plast Reconstr Surg 1996 98: 827-33.

28. Lopes AA, Port FK. The low birth hypothesis as a plausible explanation for the black/white differences in hypertension, non-insulin dependent diabetes and end-stage renal disease. AmJ Kidney Dis 1995; 25: 350-56.

29. Lopes AA. The joint effects of race and age on the risk of endstage renal disease. Tese de Doutorado, Universidade de Michigan, Estados Unidos, 1994.

30. HinchliffeSA, Lynch MRJ , Sargent PH, Howard CV, Velzen DV. The effect of intrauterinegrowth retardation on the devel opment of renal nephrons. Br J Obstet Gynaecol 1992; 99: 296-301.

31. Meyer TW, Anderson S, Rennke HG, Brenner BM. Reversing glomerular hypertension stabilizes established glomerular injury in renal ablation. J Hypertens 1986; 4: S239-S241

32. Fogo A, Ichikawa I. Evidence for a pathogenic linkage between glomerular hypertrophy and sclerosis. AmJ Kidney Dis 1991; 17: 666-69

33. Prata A, Schroeder S. A comparison of whites and negroes infected with Schistosoma mansoni in a hyperendemic area. Gaz Med Bahia 1967; 67: 93-98

34. Bina J C, Tavares-Neto J, Prata A, Azevedo ES. Greater resistance to devel opment of severe schistosomiasis in BraziIian Negroes. Hum Biol 1978; 50:41-49.

35. Freitas O. Doenças africanas no Brasil. Companhia Editora Nacional, São Paulo, SP, 1935

36. Lopes AA. Raça e Incidência de Doença Renal Terminal em Pacientes com Glomerulonefrites Primárias. Tese de Livre Docência, Universidade Federal da Bahia, 1997 